\title{
2D Quasi-Static Accurate Solutions for Isotropic Thermoelastic Materials with Applications
}

\author{
Wen-Ying Xiao, ${ }^{1}$ Jie Tong $\mathbb{D},{ }^{1,2}$ Ying-Jie Liu, ${ }^{1}$ Jiang Su, ${ }^{1}$ and Jian-Ping $\mathrm{Li}^{1}$ \\ ${ }^{1}$ School of Mechanical and Vehicle, Guangdong Polytechnic of Science and Technology, Zhuhai 519090, China \\ ${ }^{2}$ Department of Engineering Mechanics, Hunan University, Changsha 410082, China \\ Correspondence should be addressed to Jie Tong; tj8508@163.com
}

Received 9 September 2020; Revised 23 March 2021; Accepted 17 April 2021; Published 28 April 2021

Academic Editor: Vasilios Spitas

Copyright (C) 2021 Wen-Ying Xiao et al. This is an open access article distributed under the Creative Commons Attribution License, which permits unrestricted use, distribution, and reproduction in any medium, provided the original work is properly cited.

\begin{abstract}
Thermally induced stress is an important scientific problem in engineering applications. In this paper, an accurate and efficient method for the two-dimensional quasi-static thermal elastic problem is established to explore the thermal stress problem. First, the compact quasi-static two-dimensional general solution is derived in terms of simple potential functions. The general solution is simple in form and can be derived for arbitrary boundary problems subjected to a line heat load. This is completely new to the literature. Second, Green's function solutions of an infinite plane under the line pulse heat source based on the general solutions are presented to analyze the thermal stress field. Lastly, numerical results are taken into account to study the temperature and stress field induced by the dynamic heat source load. The corresponding analysis can constitute to reveal the mechanism of thermal elastic problems and provide some guidance for experiments or engineering structural design in the future work.
\end{abstract}

\section{Introduction}

Thermal stress occurs when the temperature changes for structures [1]. The applications of thermoelastic mechanics have great significance in engineering. The stiffness and strength problems caused by thermal stress are indescribably important in some engineering structures which has been an important factor for the design of engineering devices in the field of aerospace, mechanical, civil, electronic engineering, and some microstructures [2-6]. Thermal stresses are generated during high-temperature curing of the material, which induces tensile and shear stress in the structure. These stresses may cause cracks during the heat conduction process, especially during the thermal shock process $[5,6]$. The thermodynamic analysis is very important to reveal the interaction mechanism between the thermal source and structures [7-10].

Many scholars have done excellent work in thermodynamics [11-14]. Goodier proposed the integration expression of thermoelastic equations in 1937, which laid the foundation for the study of thermodynamics [11]. Boley and Weiner explored the thermal stress problems in curve beams, rings, trusses, frames, and built-up structures [15]. In 1961, Mindlin derived the governing equations for thermal media from the fundamental laws of continuum mechanics and thermodynamics [16]. Nowinski proved the laws of uniqueness for the governing equations [17]. Hetnarski introduced the thermodynamic theory systematically [13]. Therefore, it is true to use these classical elasticity theories to solve many basic thermoelastic problems. In recent years, many research studies on the thermal elastic problem can be found in the literature. Numerical method and analytical method are used to study the thermoelastic problems. The finite element method (FEM) and boundary element method (BEM) are often preferred to analyze the thermoelastic problems. For example, a thermal finite element model for the multicomponent system of high-speed metal cutting was presented [18]. The thermal stress and local damage of the composite were simulated by finite element and discrete element coupling methods [19], but it is hard to obtain the accurate solutions of the thermal elastic structure, especially near the heat source. The reason is that extremely high-gradient 
coupling field near the heat source requires a high-density grid, which usually causes ill-conditioned equations in the calculation. Thus, the calculation cannot converge to obtain an exact solution of the coupling field naturally by the traditional software platforms.

The main analytical methods are the integral transformation method, complex variable method, and classical general solution method. Integral transformation is a common method to solve differential equations. The Laplace and Hankel transform techniques have been used for the transversely isotropic thermoelastic thin plate [20]. The method of Fourier transformation is used to obtain an analytical expansion for the temperature and thermal stresses [21]. In the context of generalized theory of thermoelasticity proposed by Green and Naghdi, the thermal stress and temperature fields are obtained by using Fourier transforms [22, 23]. Lata and Parveen investigated the two-dimensional deformation of a homogeneous isotropic thermoelastic solid using the same method [24-26]. For quasi-static thermal conduction problems, three-dimensional transient heat conduction analysis was conducted by using the Laplace transformation method [27]. The thermoelasticity problem for the thick-walled cylinder subjected to transient thermal loading was analytically studied [28]. With the complexity of the structure, the integral matrix becomes larger and less efficient. And the integral transformation condition is very harsh in many cases which is difficult to get accurate results by inverse transformation. For using the complex variable method, Hasebe et al. presented thermal stresses and temperature distribution for an infinite plate with circular holes and cracks under uniform heat flux in the arbitrary direction [29]. Gao et al. presented an exact solution for the problem of an elliptical hole or a crack in a thermo-piezoelectric material [30]. Mohammad studied thermal stress analysis of the orthotropic plate containing a rectangular hole using the complex variable method [31], but complex function methods are usually only applicable to solve plane problems, which is very difficult to obtain a simple form.

The general solution method is a basic method to solve mechanical problems. It directly starts from the governing equations of mechanical models to find the general solution of partial differential equations and then combines the boundary conditions of specific problems to find the specific solution. The general solution under point loading is called Green's function solution. Green's function is an efficient tool for refined analysis of thermal stress of solid structures which can provide necessary preconditions to many highprecision numerical methods, such as boundary element method $[18,19]$. For isotropic materials, some scholars gave the basic solution of the famous closed form [32-34]. Hou's team did some work on Green's function of thermal elastic structure based on the general solutions [35-38], but the thermal elastic analysis of the isotropic structure under dynamic heat load has not been seen. In this literature, we would introduce how to get a compact and complete thermoelastic general solution for quasi-static heat conduction problems.

In this background, a harmonic function and a function which satisfies the quasi-static heat conduction equation for Green's functions under the quasi-static line heat source will be constructed in this study. Firstly, we use the differential operator theory to derive three general solutions. Secondly, by virtue of Almansi's theorem, the three general solutions can be transformed to the general solution which is expressed in terms of two harmonic functions and a function which satisfies the quasi-static heat conduction equation, respectively. Thirdly, the integral general solution expressed in one harmonic function and one function which satisfies the quasi-static heat conduction equation is obtained. As an example, the suitable functions for a quasi-static line heat source in the interior of the infinite steel plane are constructed, and the corresponding Green's solutions are presented by virtue of the obtained general solutions. In this literature, all these harmonic functions are in the form of elementary functions, and all the thermal stress components are presented in a closed form at an arbitrary point in a two-phase infinite body. The fundamental solutions for the arbitrary distributed heat source are obtained by numerical integration using Green's function under the point heat source. The method proposed in this study presents an accurate and efficient tool to analyze the thermal elastic problems, which have great engineering application value.

\section{Two-Dimensional Governing Equations for the Isotropic Thermoelastic Material}

If all components are independent of coordinate $y$ in Cartesian coordinates $(x, y, z)$, one will obtain the so-called two-dimensional problem or plane problem in two-dimensional Cartesian coordinates $(x, z)$. In this case, the corresponding constitutive equations of isotropic thermoelastic materials are in the form of

$$
\begin{aligned}
\sigma_{x} & =(\lambda+2 G) \frac{\partial u}{\partial x}+\lambda \frac{\partial \omega}{\partial z}-\alpha \theta \\
\sigma_{z} & =\lambda \frac{\partial u}{\partial x}+(\lambda+2 G) \frac{\partial \omega}{\partial z}-\alpha \theta \\
\tau_{z x} & =G\left(\frac{\partial u}{\partial z}+\frac{\partial \omega}{\partial x}\right)
\end{aligned}
$$

where $u$ and $\omega$ are the components of the mechanical displacement in $x$ - and $z$-directions, respectively, $\sigma_{x}, \sigma_{z}$, and $\tau_{z x}$ are the components of stress, $\theta$ is the temperature increment, and $\alpha$ is the thermal modulus. $\lambda$ and $G$ are the Lame constant and shear modulus, which are in the following forms for the plane stress problem and plane strain problem, respectively.

For the plane stress problem,

$$
\begin{gathered}
\lambda=\frac{\nu E}{1-v^{2}}, \\
G=\frac{E}{2(1+\nu)} .
\end{gathered}
$$

For the plane strain problem, 


$$
\begin{aligned}
& \lambda=\frac{\nu E}{(1+\nu)(1-2 v)}, \\
& G=\frac{E}{2(1+\nu)},
\end{aligned}
$$

where $E$ and $v$ are Young's modulus and Poisson's ratio of the material.

In the absence of body forces, the mechanical equilibrium equations are

$$
\begin{aligned}
& \frac{\partial \sigma_{x}}{\partial x}+\frac{\partial \tau_{z x}}{\partial z}=0 \\
& \frac{\partial \tau_{z x}}{\partial x}+\frac{\partial \sigma_{z}}{\partial z}=0
\end{aligned}
$$

The two-dimensional quasi-static heat conduction equation is

$$
\left(\frac{\partial^{2}}{\partial x^{2}}+\frac{\partial^{2}}{\partial z^{2}}\right) \theta=\frac{1}{\kappa} \frac{\partial \theta}{\partial t}
$$

where $\kappa$ is the thermal diffusivity of heat conduction:

$$
\kappa=\frac{\beta}{\rho c_{e}},
$$

where $\beta, \rho$, and $c_{e}$ are the coefficient of thermal conductivity, density, and specific heat of the material, respectively.

\section{Two-Dimensional Quasi-Static General Solutions for the Isotropic Thermoelastic Material}

In this section, the quasi-static general solutions are derived using the governing equations 1-(5) for the isotropic thermal elastic materials by virtue of the differential operator theory. Firstly, the equilibrium equations by substituting equation (1) into (3) yield

$$
\begin{aligned}
& {\left[(\lambda+2 G) \frac{\partial^{2}}{\partial x^{2}}+G \frac{\partial^{2}}{\partial z^{2}}\right] u+(\lambda+G) \frac{\partial^{2} \omega}{\partial x \partial z}-\alpha \frac{\partial \theta}{\partial x}=0} \\
& (\lambda+G) \frac{\partial^{2} u}{\partial x \partial z}+\left[G \frac{\partial^{2}}{\partial x^{2}}+(\lambda+2 G) \frac{\partial^{2}}{\partial z^{2}}\right] \omega-\alpha \frac{\partial \theta}{\partial z}=0 .
\end{aligned}
$$

A combination of the results of equations (4), (6a), and (6b) gives

$$
\mathbf{D}\left\{\begin{array}{l}
u \\
\omega \\
\theta
\end{array}\right\}=0,
$$

where $\mathbf{D}$ is the differential operator matrix defined by

$$
\mathbf{D}=\left[\begin{array}{ccc}
(\lambda+2 G) \frac{\partial^{2}}{\partial x^{2}}+G \frac{\partial^{2}}{\partial z^{2}} & (\lambda+G) \frac{\partial^{2}}{\partial x \partial z} & -\alpha \frac{\partial}{\partial x} \\
(\lambda+G) \frac{\partial^{2}}{\partial x \partial z} & G \frac{\partial^{2}}{\partial x^{2}}+(\lambda+2 G) \frac{\partial^{2}}{\partial z^{2}} & -\alpha \frac{\partial}{\partial z} \\
0 & 0 & \frac{\partial^{2}}{\partial x^{2}}+\frac{\partial^{2}}{\partial z^{2}}-\frac{1}{\kappa} \frac{\partial}{\partial t}
\end{array}\right]
$$

Based on the differential operator theory, three general solutions of homogeneous differential equation (7) can be obtained as follows:

$$
\begin{aligned}
& u=A_{i 1} f, \\
& \omega=A_{i 2} f, \\
& \theta=A_{i 3} f \quad(i=1,2,3) .
\end{aligned}
$$

$A_{i j}(i, j=1,2,3)$ are the algebraic cofactors of operator matrix D. $i, j=1,2,3$ represent row $i$ and column $j$ of operator matrix $\mathbf{D}$, and $i=1,2,3$ indicates three cases in which row $i=1$ or row $i=2$ or row $i=3$. The function $f$ should satisfy the following homogeneous equation:

$$
|\mathbf{D}| f=0 .
$$

The determinant of derivative operator matrix $\mathbf{D}$ is

$$
|\mathbf{D}|=G(\lambda+2 G)\left(\frac{\partial^{2}}{\partial x^{2}}+\frac{\partial^{2}}{\partial z^{2}}\right)^{2}\left(\frac{\partial^{2}}{\partial x^{2}}+\frac{\partial^{2}}{\partial z^{2}}-\frac{1}{\kappa} \frac{\partial}{\partial t}\right)
$$

where $A_{i j}(i, j=1,2,3)$ in equation (9) are the algebraic complements of matrix $\mathbf{D}$ which are as follows: 


$$
\begin{aligned}
& A_{11}=\left[G \frac{\partial^{2}}{\partial x^{2}}+(\lambda+2 G) \frac{\partial^{2}}{\partial z^{2}}\right]\left(\frac{\partial^{2}}{\partial x^{2}}+\frac{\partial^{2}}{\partial z^{2}}-\frac{1}{\kappa} \frac{\partial}{\partial t}\right) \\
& A_{12}=-(\lambda+G) \frac{\partial^{2}}{\partial x \partial z}\left(\frac{\partial^{2}}{\partial x^{2}}+\frac{\partial^{2}}{\partial z^{2}}-\frac{1}{\kappa} \frac{\partial}{\partial t}\right), \\
& A_{13}=0, \\
& A_{21}=-(\lambda+G) \frac{\partial^{2}}{\partial x \partial z}\left(\frac{\partial^{2}}{\partial x^{2}}+\frac{\partial^{2}}{\partial z^{2}}-\frac{1}{\kappa} \frac{\partial}{\partial t}\right), \\
& A_{22}=\left[(\lambda+2 G) \frac{\partial^{2}}{\partial x^{2}}+G \frac{\partial^{2}}{\partial z^{2}}\right]\left(\frac{\partial^{2}}{\partial x^{2}}+\frac{\partial^{2}}{\partial z^{2}}-\frac{1}{\kappa} \frac{\partial}{\partial t}\right), \\
& A_{23}=0, \\
& A_{31}=\alpha G \frac{\partial}{\partial x}\left(\frac{\partial^{2}}{\partial x^{2}}+\frac{\partial^{2}}{\partial z^{2}}\right), \\
& A_{32}=\alpha G \frac{\partial}{\partial z}\left(\frac{\partial^{2}}{\partial x^{2}}+\frac{\partial^{2}}{\partial z^{2}}\right), \\
& A_{33}=G(\lambda+2 G)\left(\frac{\partial^{2}}{\partial x^{2}}+\frac{\partial^{2}}{\partial z^{2}}\right) .
\end{aligned}
$$

When $i=1,2$ and $A_{i 3}=0$, we can obtain the general solutions in the isothermal case. The solution is the case $i=3$ considering the variable temperature. In this paper, the case $i=3$ is analyzed.

By virtue of Almansi's theorem, the function $f$ in equation (10) can be expressed in the following form:

$$
f=f_{1}+z f_{2}+f_{3}
$$

where $f_{j}$ satisfy the following harmonic equation:

$$
\begin{aligned}
\left(\frac{\partial^{2}}{\partial x^{2}}+\frac{\partial^{2}}{\partial z^{2}}\right) f_{j} & =0, \quad(j=1,2), \\
\left(\frac{\partial^{2}}{\partial x^{2}}+\frac{\partial^{2}}{\partial z^{2}}-\frac{1}{\kappa} \frac{\partial}{\partial t}\right) f_{3} & =0 .
\end{aligned}
$$

For the convenience of operation, let

$$
\begin{aligned}
& F_{1}=2 \frac{\partial f_{2}}{\partial z} \\
& F_{2}=\left(\frac{\partial^{2}}{\partial x^{2}}+\frac{\partial^{2}}{\partial z^{2}}\right) f_{3}=\frac{1}{\kappa} \frac{\partial f_{3}}{\partial t},
\end{aligned}
$$

and then

$$
\left(\frac{\partial^{2}}{\partial x^{2}}+\frac{\partial^{2}}{\partial z^{2}}\right) f=\left(\frac{\partial^{2}}{\partial x^{2}}+\frac{\partial^{2}}{\partial z^{2}}\right)\left(f_{1}+z f_{2}+f_{3}\right)=F_{1}+F_{2}
$$

Based on equations (14a)-(15), one can find that the functions $F_{j}(j=1,2)$ in equation (16) still satisfy the following harmonic equation:

$$
\begin{array}{r}
\left(\frac{\partial^{2}}{\partial x^{2}}+\frac{\partial^{2}}{\partial z^{2}}\right) F_{1}=0, \\
\left(\frac{\partial^{2}}{\partial x^{2}}+\frac{\partial^{2}}{\partial z^{2}}-\frac{1}{\kappa} \frac{\partial}{\partial t}\right) F_{2}=0 .
\end{array}
$$

Two general solutions are obtained in which $\theta=0$ when $i=1,2$. These solutions are in fact identical to those without the thermal effect which are not discussed here. Therefore, the solution should be taken in equation (12c) in which $i=3$. The following general solution is obtained:

$$
\begin{aligned}
& u=\alpha G\left(\frac{\partial F_{1}}{\partial x}+\frac{\partial F_{2}}{\partial x}\right) \\
& \omega=\alpha G\left(\frac{\partial F_{1}}{\partial z}+\frac{\partial F_{2}}{\partial z}\right) \\
& \theta=G(\lambda+2 G)\left(\frac{\partial^{2}}{\partial x^{2}}+\frac{\partial^{2}}{\partial z^{2}}\right) F_{2}=\frac{G(\lambda+2 G)}{\kappa} \frac{\partial F_{2}}{\partial t} .
\end{aligned}
$$

Let

$$
\begin{aligned}
& \psi_{1}=2 \alpha G^{2} F_{1}, \\
& \psi_{2}=2 \alpha G^{2} F_{2} .
\end{aligned}
$$

Based on equations (17) and (19), one can find that the functions $\psi_{j}(j=1,2)$ still satisfy the following harmonic equation:

$$
\begin{aligned}
\left(\frac{\partial^{2}}{\partial x^{2}}+\frac{\partial^{2}}{\partial z^{2}}\right) \psi_{1} & =0, \\
\left(\frac{\partial^{2}}{\partial x^{2}}+\frac{\partial^{2}}{\partial z^{2}}-\frac{1}{\kappa} \frac{\partial}{\partial t}\right) \psi_{2} & =0 .
\end{aligned}
$$

In combination with equations (18) and (20), the solutions are obtained:

$$
\begin{aligned}
& 2 G u=\frac{\partial \psi_{1}}{\partial x}+\frac{\partial \psi_{2}}{\partial x} \\
& 2 G \omega=\frac{\partial \psi_{1}}{\partial z}+\frac{\partial \psi_{2}}{\partial z} \\
& \frac{2 G}{\Gamma} \theta=\frac{\partial \psi_{2}}{\partial t}
\end{aligned}
$$

where

$$
\Gamma=\frac{\lambda+2 G}{\alpha \kappa}=\frac{(1-\nu) E}{\alpha \kappa(1+\nu)(1-2 \nu)} .
$$

Substituting general solution equation (21) into constitutive equation (1), the stress general solution can be simplified as follows: 


$$
\begin{aligned}
\sigma_{x} & =\frac{\partial^{2} \psi_{1}}{\partial x^{2}}+\frac{\partial^{2} \psi_{2}}{\partial x^{2}}-\frac{1}{\kappa} \frac{\partial \psi_{2}}{\partial t} \\
\sigma_{z} & =\frac{\partial^{2} \psi_{1}}{\partial z^{2}}+\frac{\partial^{2} \psi_{2}}{\partial z^{2}}-\frac{1}{\kappa} \frac{\partial \psi_{2}}{\partial t} \\
\tau_{z x} & =\frac{\partial^{2} \psi_{1}}{\partial x \partial z}+\frac{\partial^{2} \psi_{2}}{\partial x \partial z}
\end{aligned}
$$

The two-dimensional quasi-static general solutions for isotropic thermoelastic materials are obtained in equations (21) and (23). These general solutions are expressed in terms of two functions $\psi_{j}(j=1,2)$ in equation (20). The suitable functions $\psi_{j}(j=1,2)$ with undetermined constants should be constructed according to the configuration of the structures and the form of loadings. The undetermined constants will be determined by the corresponding boundary conditions, compatible conditions, and other requests. In addition, it is important that the function $\psi_{1}$ satisfies the harmonic equation and the function $\psi_{2}$ satisfies the quasi-static heat conduction equation. As an example, the suitable functions $\psi_{j}(j=1,2)$ under a dynamic line heat source will be constructed based on the obtained general solutions. For the arbitrary heat sources in actual engineering problems, their stress fields can be obtained based on the fundamental solutions for the line heat source using the superposition principle.

\section{Fundamental Solution for an Infinite Isotropic Thermoelastic Plane under a Pulse Line Heat Source}

Consider an infinite isotropic thermoelastic thin plane in two-dimensional Cartesian coordinates $(x, z)$ with the principle material direction parallel to the $x$ - and $z$-axis (Figure 1). A pulse line heat source $H(x, z, t)=H \delta(t)$ is applied at the origin $(0,0)$ on the $x o z$ plane. Based on general solutions (21) and (23), the coupled thermoelastic field in the infinite thermoelastic plane is derived in this section.

The following functions are introduced by the trial and error method:

$$
\begin{aligned}
& \psi_{1}=-2 \mathrm{~A}_{1} \ln r \\
& \psi_{2}=\mathrm{A}_{2} E_{i}\left(-\frac{r^{2}}{\tau}\right),
\end{aligned}
$$

where $\mathrm{A}_{j}(j=1,2)$ are arbitrary constants to be determined and

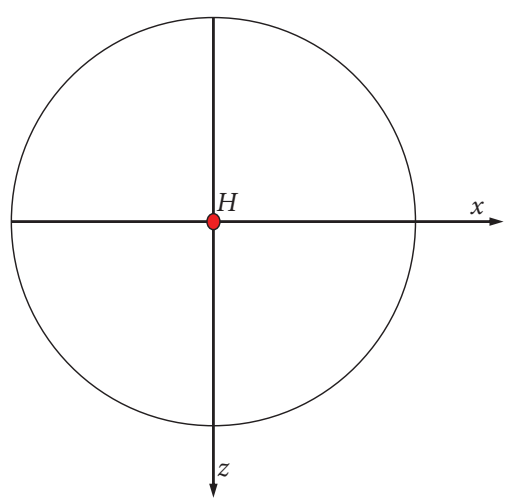

Figure 1: An infinite isotropic thermoelastic plane applied by a pulse line heat source $H$.

$$
\begin{aligned}
E_{i}(x) & =\int_{-\infty}^{x} \frac{e^{\eta}}{\eta} \mathrm{d} \eta, \\
\tau & =4 \kappa t \\
r & =\sqrt{x^{2}+z^{2}} .
\end{aligned}
$$

Substituting function (24) into general solutions (21) and (23) yields

$$
\begin{aligned}
& 2 G u_{x}=-\mathrm{A}_{1} \frac{2 x}{r^{2}}+\mathrm{A}_{2} \frac{2 x}{r^{2}} e^{-\left(r^{2} / \tau\right)}, \\
& 2 G u_{z}=-\mathrm{A}_{1} \frac{2 z}{r^{2}}+\mathrm{A}_{2} \frac{2 z}{r^{2}} e^{-\left(r^{2} / \tau\right)}, \\
& \frac{2 G}{\Gamma} \theta=-4 \kappa \mathrm{A}_{2} \frac{1}{\tau} e^{-\left(r^{2} / \tau\right)},
\end{aligned}
$$

$$
\begin{aligned}
\sigma_{x} & =-2 \mathrm{~A}_{1}\left(\frac{1}{r^{2}}-\frac{2 x^{2}}{r^{4}}\right)+2 \mathrm{~A}_{2}\left(\frac{1}{r^{2}}-\frac{2 x^{2}}{r^{4}}-\frac{2 x^{2}}{r^{2} \tau}+\frac{2}{\tau}\right) e^{-\left(r^{2} / \tau\right)}, \\
\sigma_{z} & =-2 \mathrm{~A}_{1}\left(\frac{1}{r^{2}}-\frac{2 z^{2}}{r^{4}}\right)+2 \mathrm{~A}_{2}\left(\frac{1}{r^{2}}-\frac{2 z^{2}}{r^{4}}-\frac{2 z^{2}}{r^{2} \tau}+\frac{2}{\tau}\right) e^{-\left(r^{2} / \tau\right)}, \\
\tau_{z x} & =4 x z \mathrm{~A}_{1} \frac{1}{r^{4}}-4 x z \mathrm{~A}_{2}\left(\frac{1}{r^{4}}+\frac{1}{r^{2} \tau}\right) e^{-\left(r^{2} / \tau\right)} .
\end{aligned}
$$

When the mechanical equilibrium is considered (Figure 1), the following equation can be obtained:

$$
\int_{-\infty}^{+\infty} \int_{-\infty}^{+\infty} \rho c_{e} \theta \mathrm{d} x \mathrm{~d} z=H \delta(x) \delta(z) \delta(t) .
$$

Substituting equation (26a) into (27) yields 
$-\rho c_{e} \frac{2 \mathrm{~A}_{2} \kappa \Gamma}{G} \frac{1}{\tau} \int_{-\infty}^{+\infty} \int_{-\infty}^{+\infty} \exp \left(-\frac{r^{2}}{\tau}\right) \mathrm{d} x \mathrm{~d} z=H \delta(x) \delta(z) \delta(t)$.

One useful integral is listed as follows:

$$
\begin{aligned}
\frac{1}{\tau} \int_{-\infty}^{+\infty} \int_{-\infty}^{+\infty} \exp \left(-\frac{r^{2}}{\tau}\right) \mathrm{d} x \mathrm{~d} z & =\frac{1}{\tau} \int_{-\infty}^{+\infty} \int_{-\infty}^{+\infty} \exp \left(-\frac{x^{2}+z^{2}}{\tau}\right) \mathrm{d} x \mathrm{~d} z \\
& =\int_{-\infty}^{+\infty} \int_{-\infty}^{+\infty} \exp \left(-\frac{x^{2}+z^{2}}{\tau}\right) \mathrm{d}\left(\frac{x}{\sqrt{\tau}}\right) \mathrm{d}\left(\frac{z}{\sqrt{\tau}}\right) \\
& =\left(\frac{\sqrt{\pi}}{2}\right)^{2}\left(\frac{2}{\sqrt{\pi}}\right)^{2} \int_{-\infty}^{+\infty} \exp \left(-\frac{x^{2}}{\tau}\right) \mathrm{d}\left(\frac{x}{\sqrt{\tau}}\right) \int_{-\infty}^{+\infty} \exp \left(-\frac{z^{2}}{\tau}\right) \mathrm{d}\left(\frac{z}{\sqrt{\tau}}\right)=\pi[\operatorname{erf}(\infty)]^{2}=\pi
\end{aligned}
$$

where $\operatorname{erf}(x)$ is the error function. It is defined as follows:

$$
\operatorname{erf}(x)=\frac{2}{\sqrt{\pi}} \int_{0}^{x} \exp \left(-\eta^{2}\right) \mathrm{d} \eta
$$

Substituting equation (29) into (28) yields

$$
\mathrm{A}_{2}=-\frac{H G}{2 \beta \pi \Gamma}
$$

If the time tends to infinity, all the components are given as follows:

$$
\begin{aligned}
2 G u_{x} & =-\mathrm{A}_{1} \frac{2 x}{r^{2}}+\mathrm{A}_{2} \frac{2 x}{r^{2}} \\
2 G u_{z} & =-\mathrm{A}_{1} \frac{2 z}{r^{2}}+\mathrm{A}_{2} \frac{2 z}{r^{2}} \\
\theta & =0, \\
\sigma_{x} & =-2 \mathrm{~A}_{1}\left(\frac{1}{r^{2}}-\frac{2 x^{2}}{r^{4}}\right)+2 \mathrm{~A}_{2}\left(\frac{1}{r^{2}}-\frac{2 x^{2}}{r^{4}}\right), \\
\sigma_{z} & =-2 \mathrm{~A}_{1}\left(\frac{1}{r^{2}}-\frac{2 z^{2}}{r^{4}}\right)+2 \mathrm{~A}_{2}\left(\frac{1}{r^{2}}-\frac{2 z^{2}}{r^{4}}\right), \\
\tau_{z x} & =4 x z \mathrm{~A}_{1} \frac{1}{r^{4}}-4 x z \mathrm{~A}_{2} \frac{1}{r^{4}} .
\end{aligned}
$$

The shear stress would be zero at infinity, and the following equation can be obtained:

$$
\mathrm{A}_{1}-\mathrm{A}_{2}=0
$$

Substituting equation (31) into (33) yields

$$
\mathrm{A}_{1}=\mathrm{A}_{2}=-\frac{H G}{2 \beta \pi \Gamma}
$$

Hence, Green's solution for a pulse line heat source in an infinite isotropic thermoelastic plane is determined by equation (34).

\section{Results and Discussion}

Based on the solutions derived above, we now present some numerical results. The following nondimensional components are used in the figures:

$$
\begin{aligned}
& \lambda_{1}=\frac{G}{E}=\frac{\nu}{(1+\nu)(1-2 \nu)}, \\
& G_{1}=\frac{\lambda}{E}=\frac{1}{2(1+v)}, \\
& \alpha_{1}=\frac{\alpha}{E \alpha_{0}} \text {, } \\
& E_{1}=\frac{E}{E}=1 \text {, } \\
& \Gamma_{1}=\frac{2\left(\lambda_{1}+2 G_{1}\right)}{\alpha_{1}}, \\
& \beta_{1}=\frac{\beta}{\beta}=1 \text {, } \\
& \tau^{\prime}=\frac{\tau}{r_{0}^{2}}, \\
& \kappa^{\prime}=\frac{\kappa}{4 \kappa}=\frac{1}{4}, \\
& \xi=\frac{x}{r_{0}} \\
& \zeta=\frac{z}{r_{0}} \\
& u_{k}=\frac{u_{i}}{r_{0} \alpha_{0} T_{0}}, \\
& \vartheta=\frac{\theta}{T_{0}} \\
& \sigma_{k}=\frac{\sigma_{i}}{E \alpha_{0} T_{0}}, \\
& \tau_{k l}=\frac{\tau_{i j}}{E \alpha_{0} T_{0}} \quad(i, j=x, z ; k, l=\xi, \zeta),
\end{aligned}
$$


TABLE 1: Material properties.

\begin{tabular}{lccccr}
\hline Materials & $\begin{array}{c}\text { Young's modulus } E \\
\left(10^{9} \mathrm{Nm}^{-2}\right)\end{array}$ & $\begin{array}{c}\text { Poisson's } \\
\text { ratio } \nu\end{array}$ & $\begin{array}{c}\text { Density } \rho \\
\left(10^{3} \mathrm{~kg} \cdot \mathrm{m}^{-3}\right)\end{array}$ & $\begin{array}{c}\text { Specific heat } c_{e} \\
\left(10^{3} \mathrm{~J} \cdot \mathrm{kg}^{-1}{ }^{\circ} \mathrm{C}\right)\end{array}$ & $\begin{array}{c}\text { Thermal expansion coefficient } \alpha_{0} \\
\left(10^{-6} \mathrm{~K}^{-1}\right)\end{array}$ \\
\hline Steel & 210.0 & 0.29 & 7.8 & 0.46 & 12.0 \\
\hline
\end{tabular}

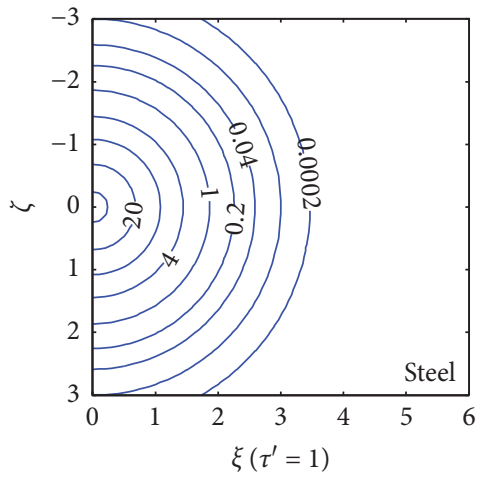

(a)

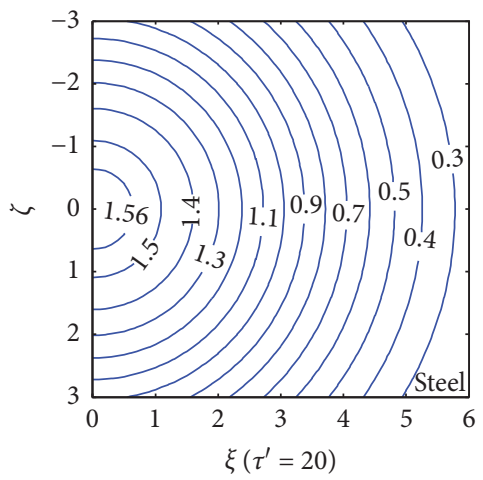

(d)

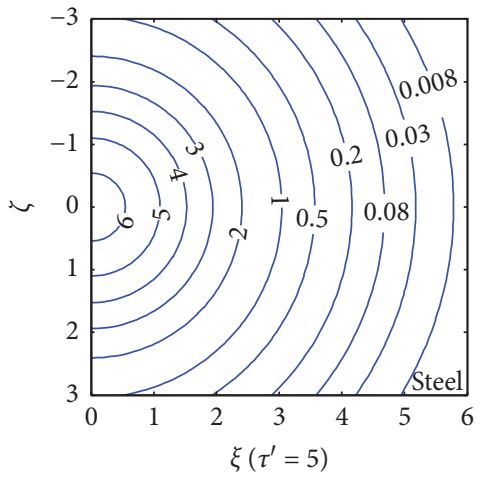

(b)

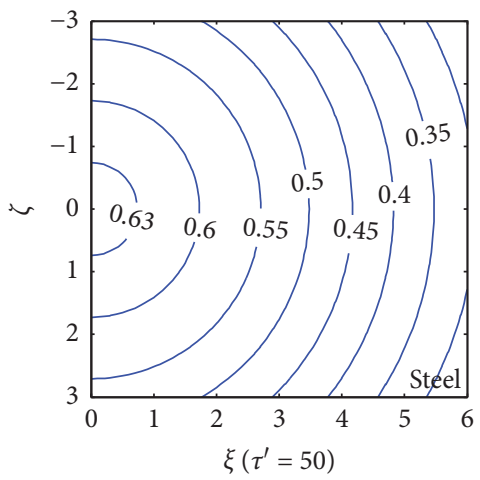

(e)

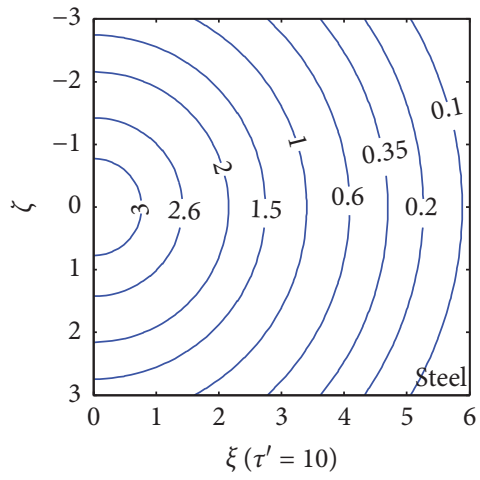

(c)

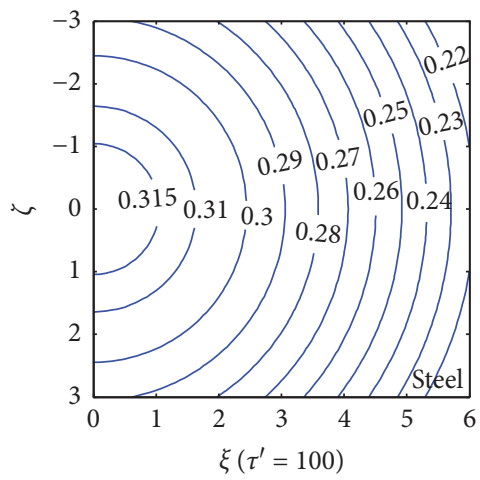

(f)

Figure 2: Temperature increment contour $\vartheta \times 10^{2}$ under a line heat source $\delta=1$.

where $r_{0}$ is a nonzero dimension and $\alpha_{0}$ and $T_{0}$ are the thermal expansion coefficient and reference temperature, respectively. Equations (32a) and (32b) should be rewritten in the following nondimensional form:

$$
\mathrm{A}_{2}=-\frac{2 \delta G}{\pi \Gamma_{1}}
$$

where $\delta$ is a nondimensional heat source given as follows:

$$
\delta=\frac{H}{r_{0}^{2} T_{0} \rho c_{e}} .
$$

Here, let $\delta=1$ act at the point $(0,0)$, so the heat source $H=r_{0}^{2} T_{0} \rho c_{e}$. The material properties for steel are listed in Table 1 .

\subsection{Thermoelastic Field Contours under the Pulse Line Heat} Source. Temperature and thermal-stress distribution contours of steel are given in Figures 2-5 when a pulse line heat source acts on the center of the plane. On the basis of the contours plotted, one can find all components are singular at the origin in which the line heat source is located. The components change gradually with time $\tau$ and then tend to be stable which do not change over time. It can be found that there is a great thermal impact near the heat source at the moment of the heat source action. So, the stresses are relatively large which change quite dramatically. With time going on, the stress values decrease gradually with the diffusion of the heat source. We can clearly see the trend of the temperature field and stress field, which helps engineers to predict the engineering structure stress state under the action of thermal load in advance.

(1) Figure 2 shows that the contours of temperature increment $\vartheta(\theta)$ in the infinite plane are all normal circles, which is because the thermal diffusivity $\kappa$ along the $x$-and $z$-axis is equal for steel, respectively. In the initial stage of the heat source acting on the plane, the heat source is confined in a small region. And the temperature increment is very small which nearly approaches to be zero, far away from the heat source region. This also indicates that the temperature gradient is very large under the pulse heat source. A large stress field is easily generated near the operating point of the heat source which could cause 


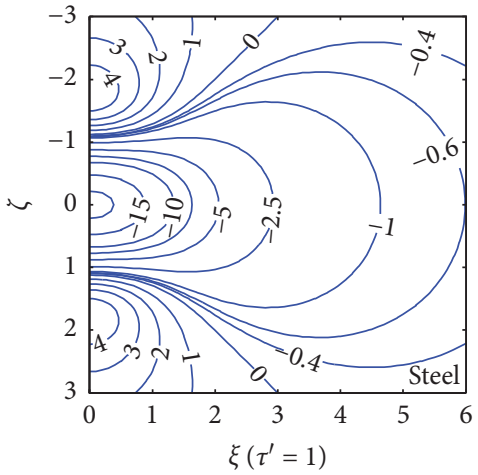

(a)

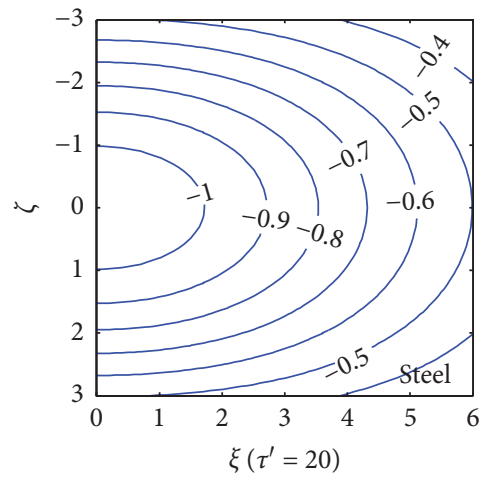

(d)

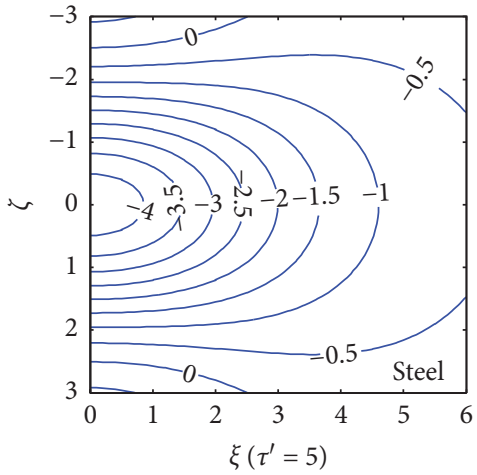

(b)

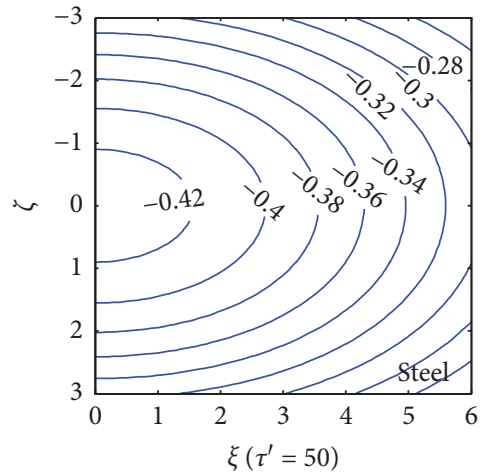

(e)

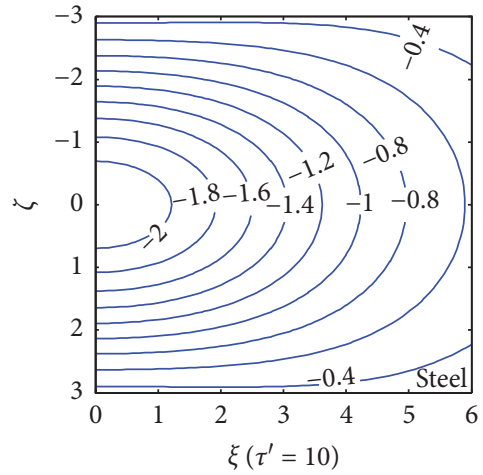

(c)

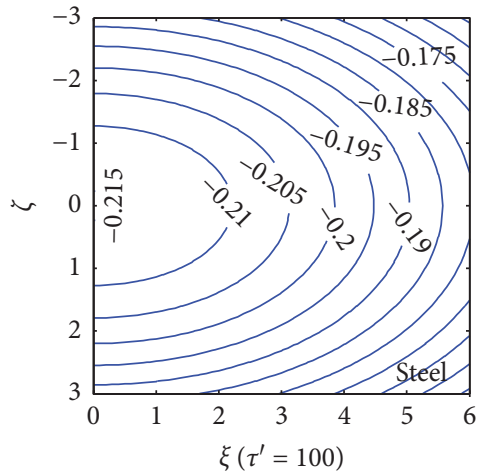

(f)

Figure 3: Stress contour $\sigma_{\xi} \times 10^{2}$ under a line heat source $\delta=1$.

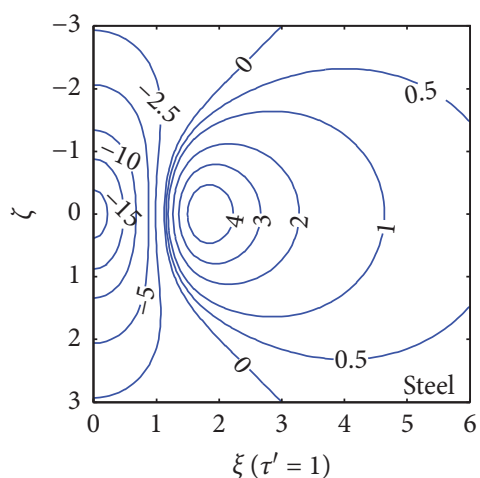

(a)

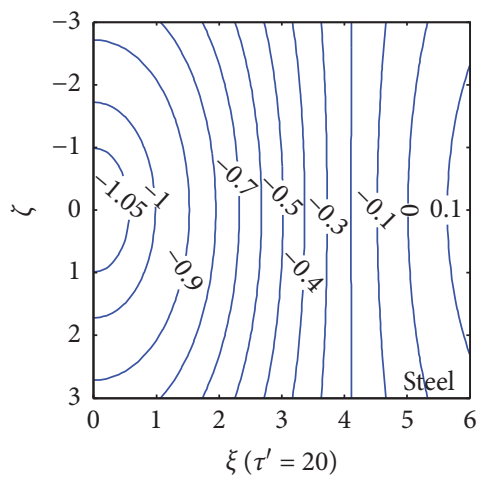

(d)

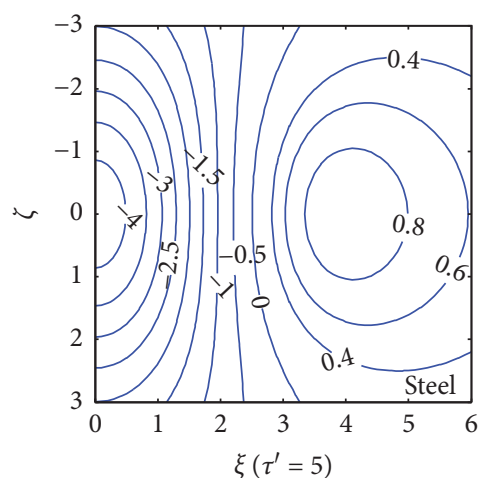

(b)

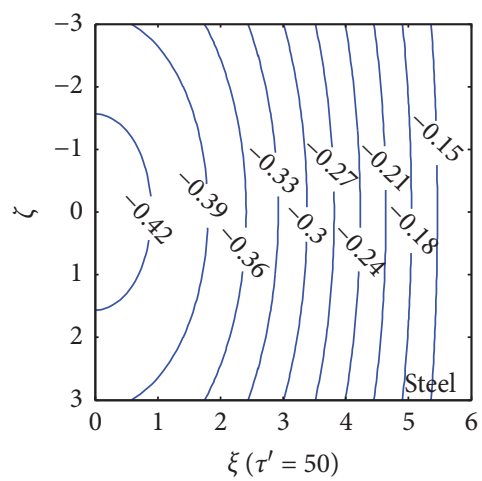

(e)

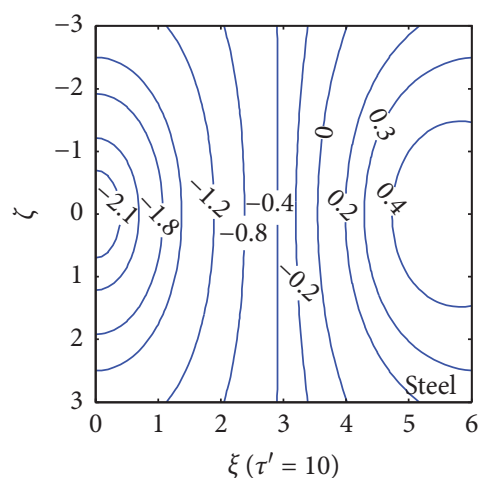

(c)

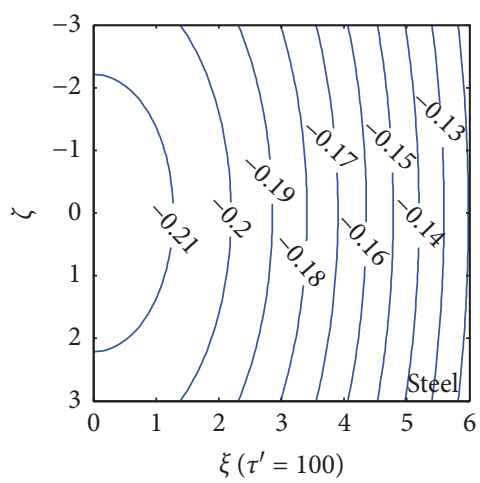

(f)

Figure 4: Stress contour $\sigma_{\zeta} \times 10^{2}$ under a line heat source $\delta=1$. 


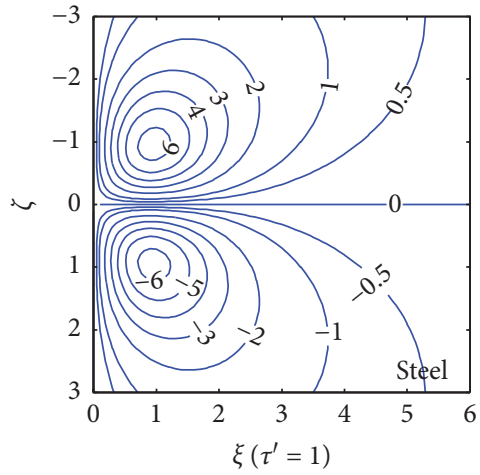

(a)

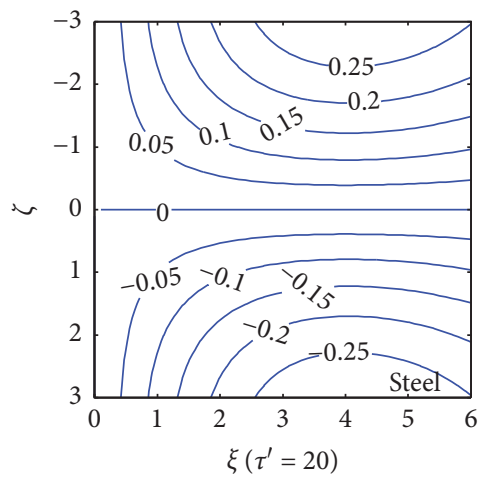

(d)

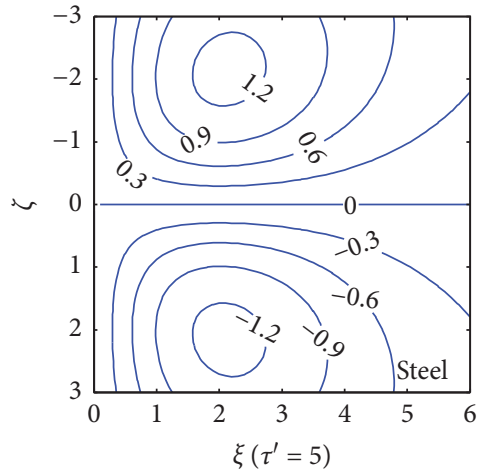

(b)

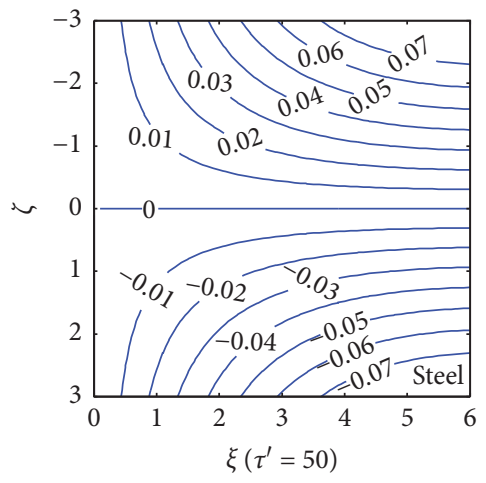

(e)

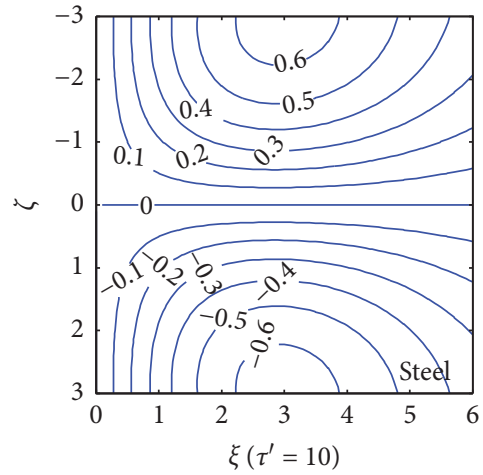

(c)

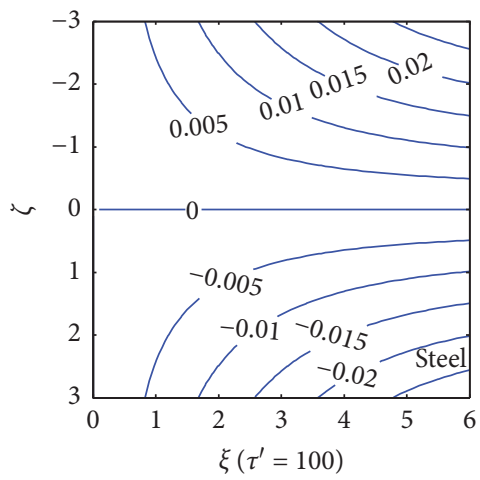

(f)

FiguRe 5: Stress contour $\tau_{\varsigma \xi} \times 10^{2}$ under a line heat source $\delta=1$.

structural damage. With the passage of time, the gradients of temperature increment decrease gradually and tend to be in a stable state which clearly reveals the change process of the temperature field.

(2) It can be seen from Figures 3 and 4 that the radial component $\sigma_{\xi}\left(\sigma_{x}\right)$ and axial component $\sigma_{\varsigma}\left(\sigma_{z}\right)$ of thermal stress have positive and negative values which vary with the heat source acting time. The gradients of negative contours for the axial stress $\sigma_{\varsigma}\left(\sigma_{z}\right)$ are much larger than those of positive contours at the time $\tau^{\prime}=1$. As time goes on, the zero contour moves outward, and the whole stress fields would be compressive stress. In the initial stage of the heat acting, the stresses rise and drop sharply because the heat energy is mainly concentrated near the heat source region.

(3) Figure 5 shows that there is a zero common tangent for shear stress $\tau_{\varsigma \xi}\left(\tau_{z x}\right)$ in an infinite steel plane. And the contours of shear stress $\tau_{\varsigma \xi}\left(\tau_{z x}\right)$ are symmetric with the zero contours. The shear stress $\tau_{\varsigma \xi}\left(\tau_{z x}\right)$ changes significantly between the interfaces of the zero contour at the beginning which means the stress failure of the materials is possible to occur at the time.

5.2. Thermoelastic Field Contours under Arbitrary Distributed Pulse Heat Source Load. In this section, we calculate the temperature and thermal stress distributions under different pulse-type heat source loads. By virtue of the superposition principle and numerical integration method, the coupled field under arbitrary distributed pulse heat source can be obtained easily. In this section, we consider one kind of heat source loaded on the plane.

For example, the dimensionless distributed pulse heat source loaded on the plane is in the form of

$$
f(\xi, \varsigma)=\xi^{2}+\varsigma^{2}, \quad(-1 \leq \xi \leq 1,-1 \leq \varsigma \leq 1) .
$$

The region of the distributed pulse heat source is

$$
A(\xi, \varsigma): \xi^{2}+\varsigma^{2}=1, \quad(-1 \leq \xi \leq 1,-1 \leq \varsigma \leq 1) .
$$

The contours of the stress components and temperature component for the distributed heat source are shown in Figures 6-9. Figures 6-9 show that the temperature and stress components tend to be stable gradually which is the same as the variation trend of that in Section 5.1.

5.3. Variation Characteristics of the Thermal Stress Field Components. The distributions of temperature and thermal stress at different positions of the plane with different times are given in Figures 10-13. Figure 10 depicts the variation of temperature distribution with $t$. It indicates the values of temperature increment $\vartheta$ have a sharp rise and then have a rapid decline when $0<t<20$. It is noticed that the temperature values slightly decrease when $t \geq 50$ and then tend 


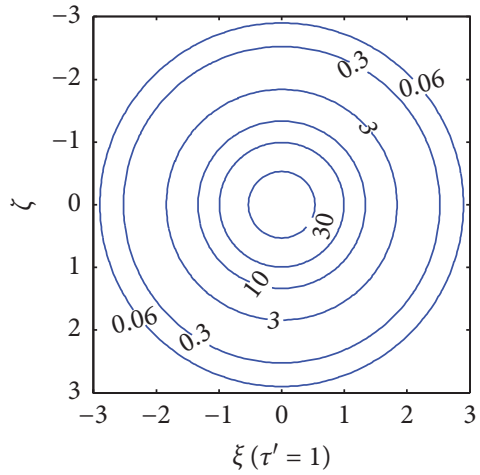

(a)

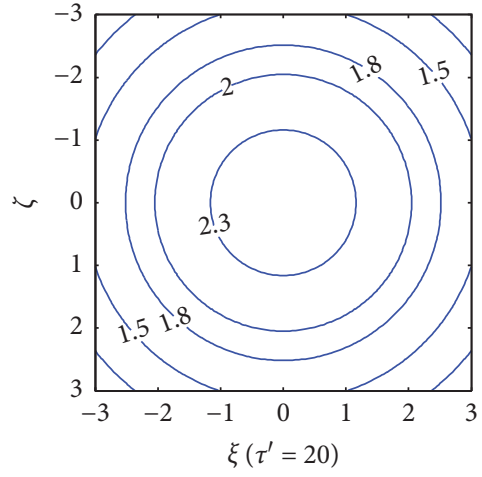

(b)

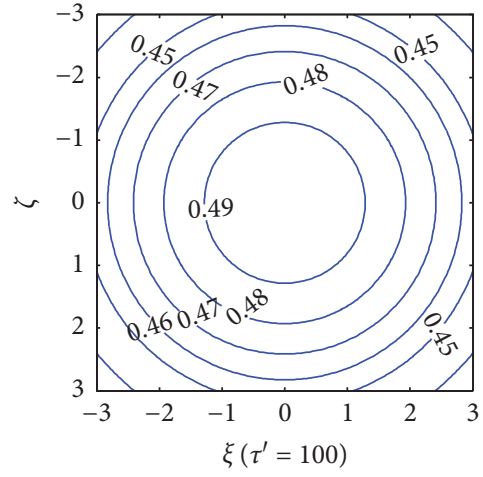

(c)

FIgURE 6: Temperature increment contour $\vartheta \times 10^{2}$ under the distributed pulse heat source.

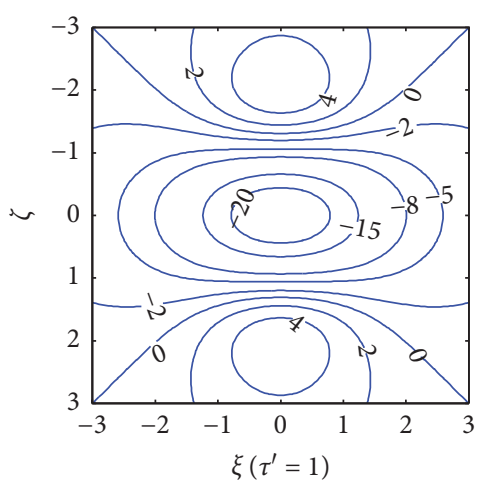

(a)

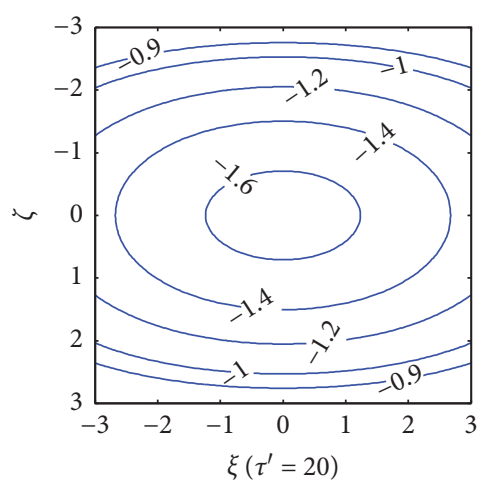

(b)

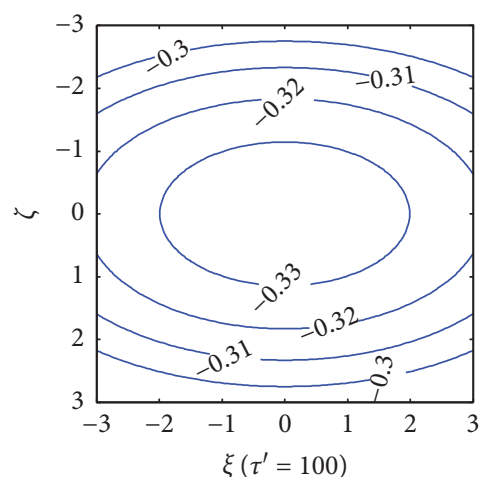

(c)

FIGURE 7: Stress contour $\sigma_{\xi} \times 10^{2}$ under the distributed pulse heat source.

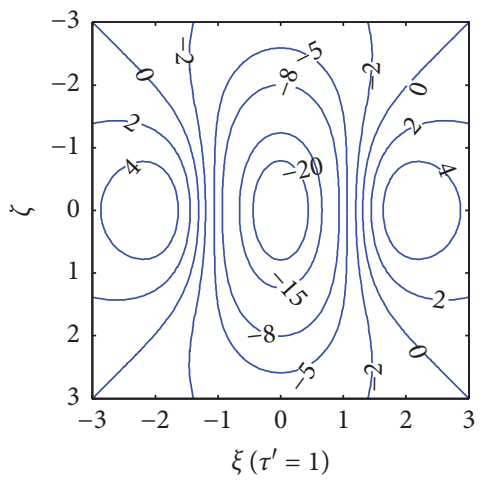

(a)

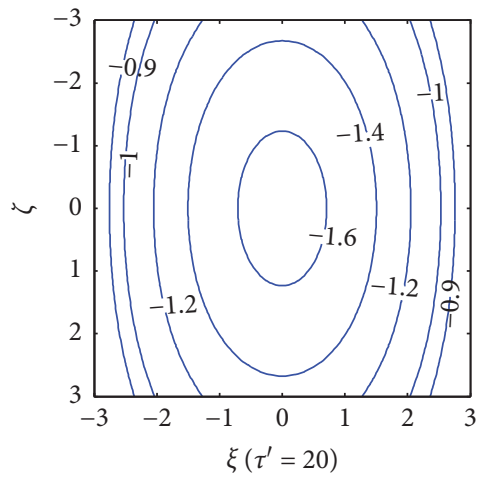

(b)

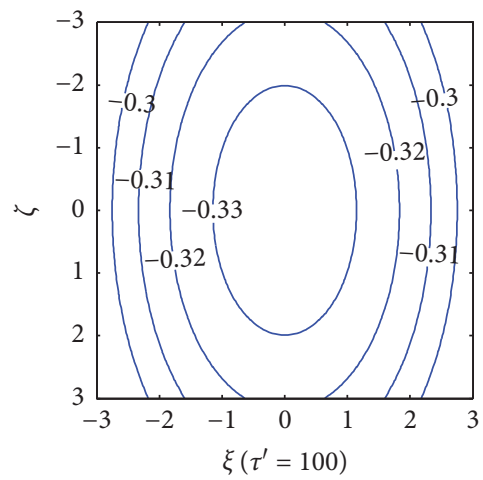

(c)

FIGURE 8: Stress contour $\sigma_{\zeta} \times 10^{2}$ under the distributed pulse heat source. 


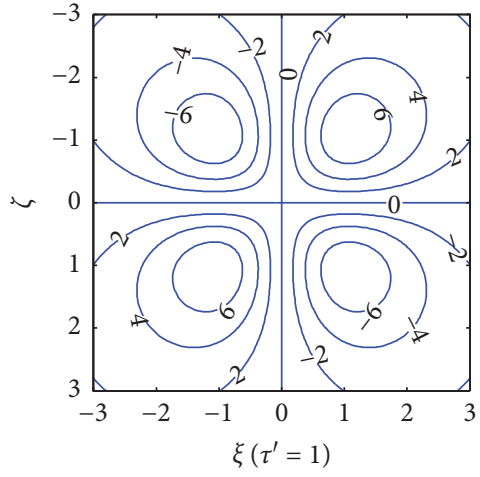

(a)

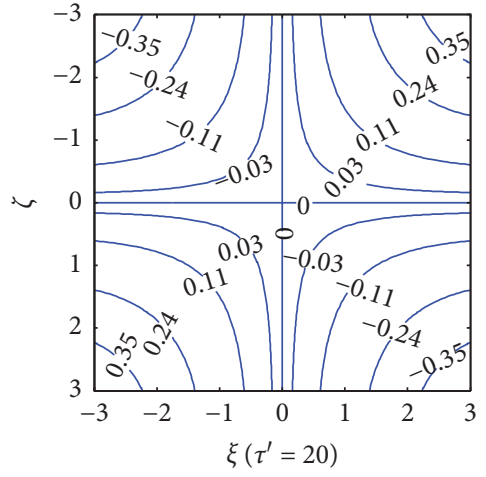

(b)

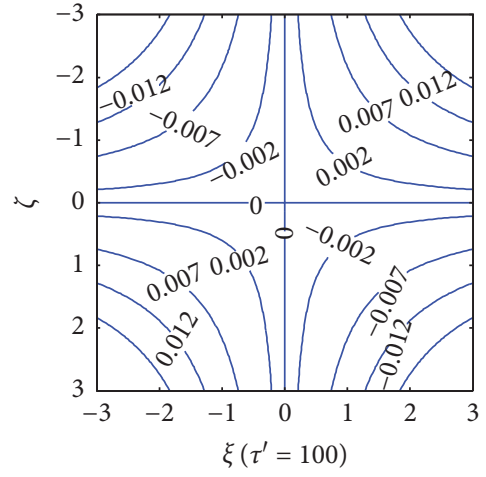

(c)

FIGURE 9: Stress contour $\tau_{\varsigma \xi} \times 10^{2}$ under the distributed pulse heat source.

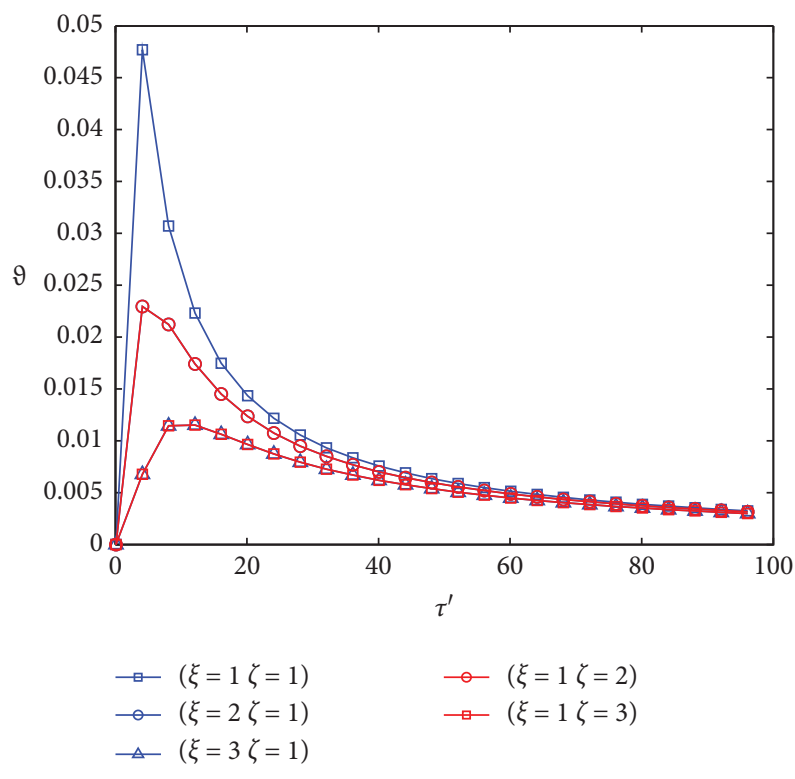

FIgURE 10: Temperature distributions at different locations.

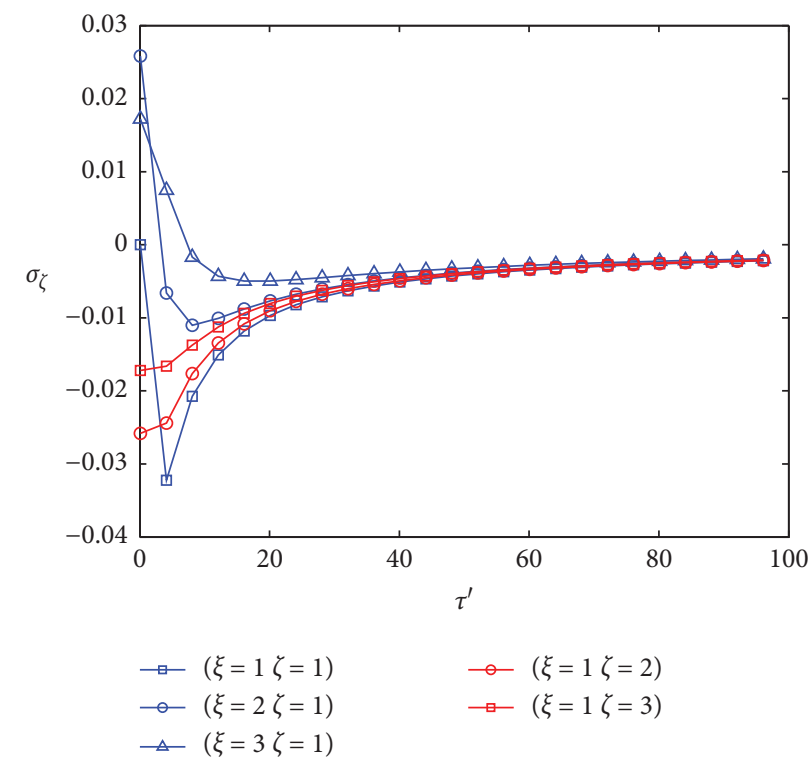

FIgURE 11: Radial component of thermal stress distributions at different locations. 


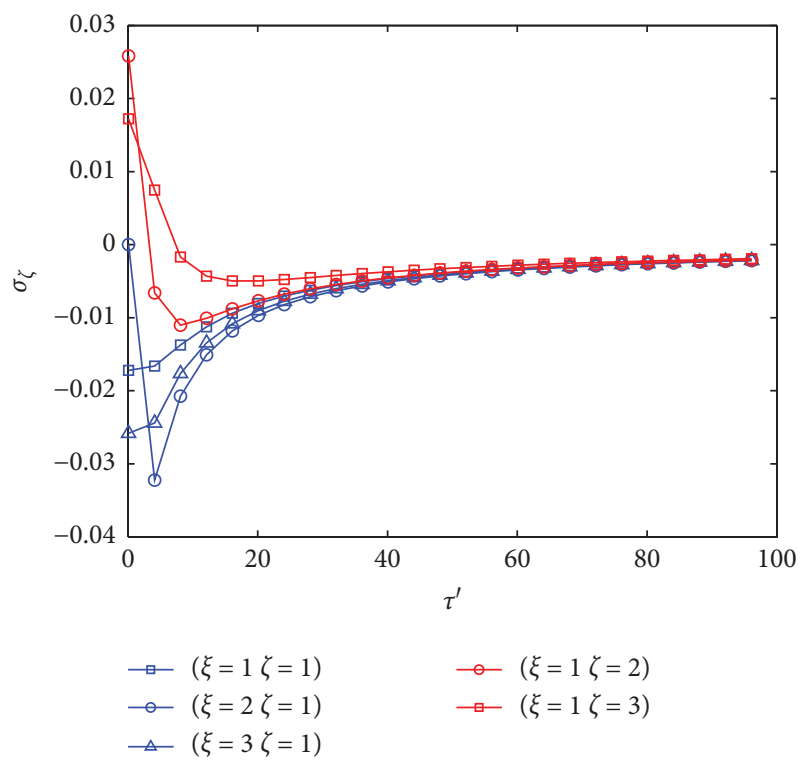

Figure 12: Axial component of thermal stress distributions at different locations.

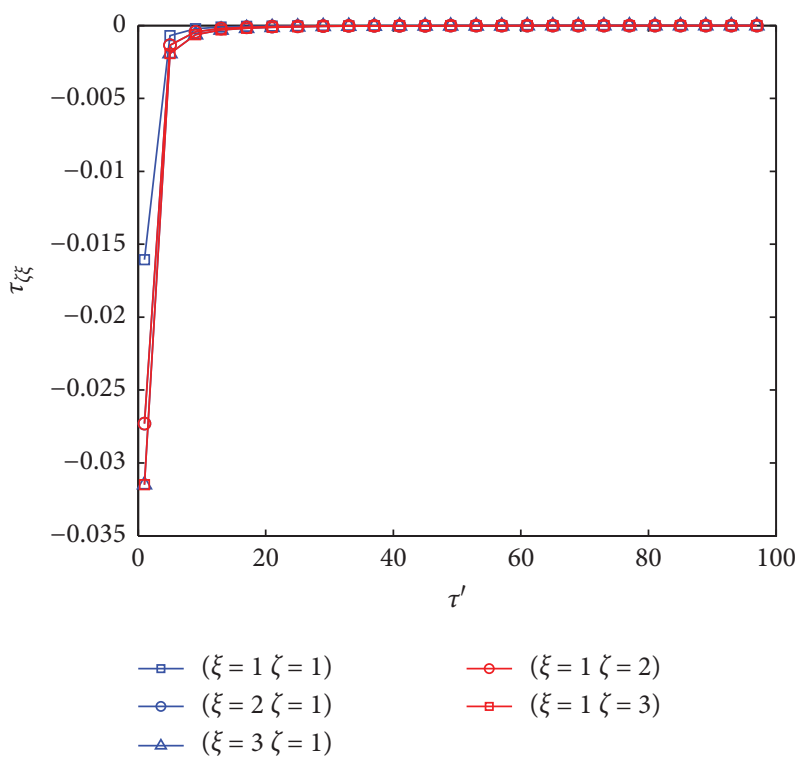

FIGURE 13: Shear stress component of thermal stress distributions at different locations.

to be stable. Figures 11 and 12 exhibit the variations of radial and axial stress components with time $t$. The thermal stresses show compressive stress when they are stable. Figure 13 shows the variation of the shear stress with time $t$. It is noticed that the values of the shear stress $\tau_{\xi \zeta}$ become stable when $t>20$. When $0<t<20$, the shear stress values are negative and increase rapidly.

\section{Conclusions}

In this paper, the compact quasi-static two-dimensional general solution for isotropic thermoelastic materials is presented by virtue of differential operator theory and
Almansi's theorem. The general solutions are expressed using simple explicit functions. Based on the general solutions, Green's functions are obtained for a line heat source. Also, all Green's components are expressed in simple functions, which are very convenient to use. The solution in this paper is obtained completely from the governing equation. No simplifying assumptions have been made. As an example, Green's solutions for a pulse line heat source in the interior of an infinite steel plane are presented by virtue of the obtained general solution. Some numerical analyses are taken into account to study the distribution of the thermoelastic field under the action of the dynamic heat source. The numerical results of this study can give some 
theoretical basis for revealing the mechanism of the thermal elastic problem and provide some guidance for experiments or engineering structural design in the future work.

\section{Data Availability}

All the data generated or analyzed during this study are included within this article.

\section{Additional Points}

Highlights. (1) A 2D quasi-static isotropic thermoelastic model for thermal fields was established. (2) Green's function is expressed in terms of concise elementary functions. (3) The accurate thermal stress is analyzed.

\section{Conflicts of Interest}

The authors declare that they have no conflicts of interest.

\section{Acknowledgments}

The authors thankfully acknowledge the financial support from the Guangzhou Science, Technology and Innovation Commission of China (no. 201904010204), the Hunan Province Natural Science Foundation of China (no. 2019JJ50633), the Department of Education of Guangdong Province of China (nos. 2018GKTSCX107, 2019GKTSCX031, and 2019GKTSCX032), and the Guangdong Province Natural Science Foundation of China (no. 2017A030310578).

\section{References}

[1] M. Nemat-Alla, "Reduction of thermal stresses by developing two-dimensional functionally graded materials," International Journal of Solids and Structures, vol. 40, no. 26, pp. 7339-7356, 2003.

[2] O. U. Khan and B. S. Yilbas, "Laser heating of sheet metal and thermal stress development," Journal of Materials Processing Technology, vol. 155, pp. 2045-2050, 2004.

[3] H. Dong, J. Cui, Y. Nie, Q. Ma, and Z. Yang, "Multiscale computational method for thermoelastic problems of composite materials with orthogonal periodic configurations," Applied Mathematical Modelling, vol. 60, pp. 634-660, 2018.

[4] J. Wang, Y. Li, X. Liu, C. Shen, H. Zhang, and K. Xiong, "Recent active thermal management technologies for the development of energy-optimized aerospace vehicles in China," Chinese Journal of Aeronautics, vol. 34, no. 2, pp. 1-27, 2021.

[5] X. Yu and D. M. Leitner, "Heat flow in proteins: computation of thermal transport coefficients," The Journal of Chemical Physics, vol. 122, no. 5, Article ID 054902, 2005.

[6] S. Sunder, S. Sathi, K. Venkataramanan et al., "A rare case of type I renal tubular acidosis with membranous nephropathy presenting as hypokalemic paralysis," Case Reports in $\mathrm{Ne}$ phrology and Urology, vol. 3, no. 2, pp. 91-98, 2013.

[7] H. Song, K. Xie, and C. Gao, "Progressive thermal stress distribution around a crack under Joule heating in orthotropic materials," Applied Mathematical Modelling, vol. 86, pp. 271293, 2020.
[8] J. Kuutti and I. Virkkunen, "The effect of subsurface crack opening on the stress intensity factor under cyclic thermal loads," Engineering Fracture Mechanics, vol. 218, Article ID 106600, 2019.

[9] G. Chen, Y. Wang, J. Zhang, and J. Bi, “An analytical solution for two-dimensional modeling of repetitive long pulse laser heating material," International Journal of Heat and Mass Transfer, vol. 104, pp. 503-509, 2017.

[10] G. Chen, "Analytical solutions for temperature and thermalstress modeling of solid material induced by repetitive pulse laser irradiation," Optik, vol. 135, pp. 16-26, 2017.

[11] J. N. Goodier, "Integration of thermoelastic equations," Philosophical Magazine, vol. 231017 pages, 1937.

[12] D. M. Gilbey, "Theory of thermal stresses," Journal of the Less Common Metals, vol. 1, no. 2, pp. 139-144, 1959.

[13] R. B. Hetnarski, Encyclopedia of Thermal Stresses, Springer, Berlin, Germany, 2014.

[14] W. Nowacki, "Problems of thermoelasticity," Progress in Aerospace Sciences, vol. 10, pp. 1-63, 1970.

[15] B. A. Boley, J. H. Weiner, and W. Prager, "Theory of thermal stresses," Journal of Applied Mechanics, vol. 28, no. 2, p. 318, 1961.

[16] J. N. Sharma and P. K. Sharma, "Free vibration analysis of homogeneous transversely isotropic thermoelastic cylindrical panel," Journal of Thermal Stresses, vol. 25, no. 2, pp. 169-182, 2002.

[17] J. N. Nowinski, Theory of Thermoelasticity with Applications, Sijthoff \& Noordhoff International Publishers, Rockville, MD, USA, 1978.

[18] Q. Xia and D. R. H. Gillespie, "Quasi-static finite element modelling of thermal distribution and heat partitioning for the multi-component system of high speed metal cutting," Journal of Materials Processing Technology, vol. 275, Article ID 116389, 2020.

[19] W. Leclerc, H. Haddad, and M. Guessasma, "DEM-FEM coupling method to simulate thermally induced stresses and local damage in composite materials," International Journal of Solids and Structures, vol. 160, pp. 276-292, 2019.

[20] I. Kaur and P. Lata, "Transversely isotropic thermoelastic thin circular plate with constant and periodically varying load and heat source," International Journal of Mechanical and Materials Engineering, vol. 14, no. 1, p. 10, 2019.

[21] A. A. Yevtushenko and O. M. Ukhanska, "The thermal stresses and displacements in a two-dimensional convective halfspace for a moving heat source," International Journal of Heat and Mass Transfer, vol. 37, no. 17, pp. 2737-2743, 1994.

[22] K. L. Verma, "Thermoelastic vibrations of a transversely isotropic plate with thermal relaxations," International Journal of Solids and Structures, vol. 38, no. 46-47, pp. 8529-8546, 2001.

[23] S. H. Mallik and M. Kanoria, "A two dimensional problem for a transversely isotropic generalized thermoelastic thick plate with spatially varying heat source," European Journal of Mechanics-A/Solids, vol. 27, no. 4, pp. 607-621, 2008.

[24] P. Lata and S. Singh, "Effects of nonlocality and two temperature in a nonlocal thermoelastic solid due to ramp type heat source," Arab Journal of Basic and Applied Sciences, vol. 27, no. 1, pp. 358-364, 2020.

[25] R. Kumar, N. Sharma, and P. Lata, "Effects of Hall current in a transversely isotropic magneto thermoelastic with and without energy dissipation due to normal force," Structural Engineering and Mechanics, vol. 57, no. 1, pp. 91-103, 2016.

[26] P. Lata and S. Singh, "Thermomechanical interactions in a non local thermoelastic model with two temperature and 
memory dependent derivatives," Coupled Systems Mechanics, vol. 9, no. 5, pp. 397-410, 2020.

[27] S. Guo, J. Zhang, G. Li, and F. Zhou, "Three-dimensional transient heat conduction analysis by Laplace transformation and multiple reciprocity boundary face method," Engineering Analysis with Boundary Elements, vol. 37, no. 1, pp. 15-22, 2013.

[28] A. R. Shahani and S. M. Nabavi, "Analytical solution of the quasi-static thermoelasticity problem in a pressurized thickwalled cylinder subjected to transient thermal loading," Applied Mathematical Modelling, vol. 31, no. 9, pp. 1807-1818, 2007.

[29] H. Norio, I. Hideaki, and N. Takuji, "Stress intensity factors of cracks initiating from a rhombic hole due to uniform heat flux," Engineering Fracture Mechanics, vol. 42, no. 2, pp. 331-337, 1992.

[30] C.-F. Gao, Y.-T. Zhao, and M.-Z. Wang, “An exact and explicit treatment of an elliptic hole problem in thermopiezoelectric media," International Journal of Solids and Structures, vol. 39, no. 9, pp. 2665-2685, 2002.

[31] M. Jafari and M. Jafari, "Thermal stress analysis of orthotropic plate containing a rectangular hole using complex variable method," European Journal of Mechanics-A/Solids, vol. 73, pp. 212-223, 2019.

[32] Z. Xu, M. Chen, C. Su, X. Fan, and Z. Guan, "Dynamic analysis of multi-crack problems by the spline fictitious boundary element method based on Erdogan fundamental solutions," Engineering Analysis with Boundary Elements, vol. 107, pp. 33-46, 2019.

[33] P. S. Dineva, G. D. Manolis, and F. Wuttke, "Fundamental solutions in 3D elastodynamics for the BEM: a review," Engineering Analysis with Boundary Elements, vol. 105, pp. $47-69,2019$.

[34] O. Lafe, "Boundary element methods in engineering science," International Journal for Numerical and Analytical Methods in Geomechanics, vol. 7, no. 2, p. 286, 1983.

[35] P.-F. Hou, S. He, and C.-P. Chen, "2D general solution and fundamental solution for orthotropic thermoelastic materials," Engineering Analysis with Boundary Elements, vol. 35, no. 1, pp. 56-60, 2011.

[36] P.-F. Hou, Q.-H. Li, and H.-Y. Jiang, "Three-dimensional steady-state general solution for isotropic thermoelastic materials with applications II: Green's functions for twophase infinite body," Journal of Thermal Stresses, vol. 36, no. 8, pp. 851-867, 2013.

[37] P.-F. Hou, J. Tong, and M. Zhao, "Two-dimensional steadystate general solution for isotropic thermoelastic materials with applications. I: general solutions and fundamental solutions," Applied Mathematical Modelling, vol. 37, no. 23, pp. 9786-9797, 2013.

[38] Q. Li and P. Hou, “Three-dimensional Green's functions for fluid and isotropic thermoelastic solid two-phase materials under heat loading," Case Studies in Thermal Engineering, vol. 20, Article ID 100631, 2020. 